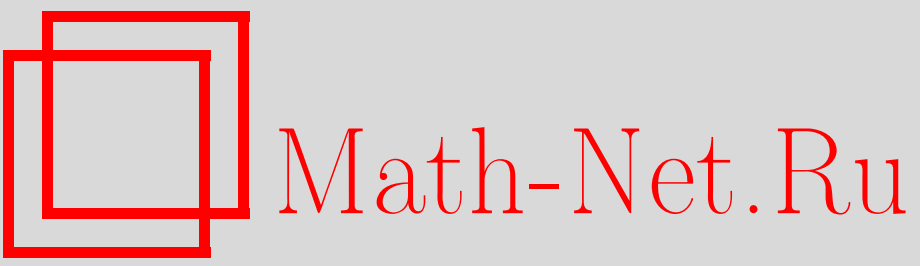

Ю. В. Афанасенкова, Ю. А. Гладышев, В. В. Калманович, Некоторые методы решения задач теплопроводности многослойной среды при наличии источников тепла, Итоги науки и техн. Сер. Соврем. мат. и ее прил. Темат. обз., 2019, том $172,3-8$

DOI: https://doi.org/10.36535/0233-6723-2019-172-3-8

Использование Общероссийского математического портала Math-Net.Ru подразумевает, что вы прочитали и согласны с пользовательским соглашением

http://www.mathnet.ru/rus/agreement

Параметры загрузки:

IP : 34.227 .88 .159

26 апреля 2023 г., 17:30:32 


\title{
НЕКОТОРЫЕ МЕТОДЫ РЕШЕНИЯ ЗАДАЧ ТЕПЛОПРОВОДНОСТИ МНОГОСЛОЙНОЙ СРЕДЫ ПРИ НАЛИЧИИ ИСТОЧНИКОВ ТЕПЛА
}

\author{
(c) 2019 г. Ю. В. АФАНАСЕНКОВА, Ю. А. ГЛАДЫШЕВ, В. В. КАЛМАНОВИЧ
}

\begin{abstract}
АннотАция. Решение задачи о фазовых переходах в многослойных средах при их нагревании представляет практический интерес ввиду все большего использования многослойных материалов в технике и строительстве при различных температурных условиях. В данной работе предполагается, что в среде присутствуют распределенные тепловые источники, вызванные физическими или химическими процессами, что может привести к фазовым переходам. В работе описаны метод обобщенных степеней Берса и матричный метод решения задачи теплопроводности в многослойной среде и поиска границ фазовых переходов.
\end{abstract}

Ключевые слова: обобщенные степени Берса, уравнение теплопроводности, задача Коши, матричный метод, многослойная среда, фазовые переходы.

\section{METHODS FOR SOLVING PROBLEMS ON THERMAL CONDUCTIVITY OF MULTILAYER MEDIA IN THE PRESENCE OF HEAT SOURCES}

\author{
(c) 2019 YU. V. AFANASENKOVA, YU. A. GLADYSHEV, V. V. KALMANOVICH
}

\begin{abstract}
The solution of problems on phase transitions in multilayer media when they are heated is of practical interest owing to the increasing use of multilayer materials in engineering and construction under various temperature conditions. In this paper, we assume that the medium contains distributed heat sources caused by physical or chemical processes, which can lead to phase transitions. We describe the method of generalized Bers degrees and the matrix method for solving the heat conduction problem in multilayer media and for the search for boundaries of phase transitions.
\end{abstract}

Keywords and phrases: generalized Bers degrees, heat equation, Cauchy problem, matrix method, multilayer medium, phase transitions.

AMS Subject Classification: 34B05, 34B60, 80A20

1. Введение. В ряде технических устройств возникает необходимость поддерживать постоянную или переменную во времени температуру в некоторой области, окруженной твердыми стенками. С этой целью внутри стенки помещают источники или, наоборот, поглотители тепла. Системы теплоизоляции в настоящее время весьма усложнились; они могут содержать и элементы подогрева стенки. Поэтому представляет интерес изучить теплопроводность стенки при наличии тепловых источников.

Первоначально рассматривается однослойная оболочка, так как переход к многослойной системе может быть осуществлен стандартными матричными методами.

При наличии распределенных источников тепла температурное поле не является монотонной функцией на рассматриваемом промежутке, а имеет на нем максимальное значение внутри слоя;

Работа выполнена при поддержке Российского фонда фундаментальных исследований (проект № 19-03-00271), а также совместного проекта Российского фонда фундаментальных исследований и правительства Калужской области № 18-41-400001. 
(1)

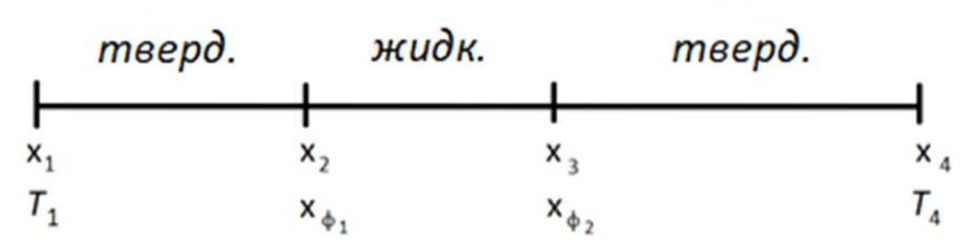

Рис. 1. Модель из трех слоев, описывающая фазовые переходы в оболочке

в результате в оболочке могут произойти фазовые переходы. В этом случае система моделируется тремя слоями (см. рис. 1).

Предположим, что температура фазового перехода $T_{\phi}$ вещества задана, причем коэффициент теплопроводности разных фаз резко различен. Координаты $x_{1}, x_{4}$ определяют внешние границы слоя, являются заданными величинами, координаты $x_{2}, x_{3}$ определяют границы фаз и являются неизвестными (искомыми) величинами. Положим, что на внешних границах слоя $x_{1}, x_{4}$ заданы соответственно внешние температуры $T_{1}, T_{4}$, меньшие $T_{\phi}$.

2. Постановка задачи и решение задачи теплопроводности в обобщенных степенях Берса. Основная система уравнений теплопроводности в многослойной среде имеет простую форму (см. [6]):

$$
\frac{d}{d x}\left(\lambda^{(i)} \frac{d T^{(i)}}{d x}\right)=-q, \quad i=1,2,3,
$$

где $\lambda^{(i)}$ - коэффициент теплопроводности, $q=$ const - мощность источников тепла, верхний индекс в скобках $(i)$ определяет номер слоя.

Основная трудность состоит в нахождении решения, удовлетворяющего условиям согласования на границах слоев

и внешним граничным условиям

$$
\begin{gathered}
\left.T^{(1)}(x)\right|_{x=x_{\phi_{1}}}=\left.T^{(2)}(x)\right|_{x=x_{\phi_{1}}},\left.\quad T^{(2)}(x)\right|_{x=x_{\phi_{2}}}=\left.T^{(3)}(x)\right|_{x=x_{\phi_{2}}}, \\
\left.\lambda^{(1)} \frac{d T^{(1)}}{d x}\right|_{x=x_{\phi_{1}}}=\left.\lambda^{(2)} \frac{d T^{(2)}}{d x}\right|_{x=x_{\phi_{1}}},\left.\quad \lambda^{(2)} \frac{d T^{(2)}}{d x}\right|_{x=x_{\phi_{2}}}=\left.\lambda^{(3)} \frac{d T^{(3)}}{d x}\right|_{x=x_{\phi_{2}}}
\end{gathered}
$$

$$
\left.T^{(1)}(x)\right|_{x=x_{1}}=T_{1},\left.\quad T^{(3)}(x)\right|_{x=x_{4}}=T_{4} .
$$

При традиционном методе решения системы (1)-(4) имеем шесть искомых параметров. Хотя есть полная уверенность в наличии единственного решения, однако их физическая интерпретация сильно затруднена большим числом параметров. Дальнейшее решение состоит в таком выборе значений $x_{\phi_{1}}, x_{\phi_{2}}$, чтобы удовлетворить условиям фазового перехода.

Представим искомое решение как сумму обобщенных степеней Берса (см. $[2,7])$, что дает возможность значительно упростить краевую задачу, сведя решение к поиску только трех коэффициентов $C_{1}, C_{2}, C_{3}$. Решение для модели трех слоев возьмем в виде

$$
\begin{array}{ll}
T^{(1)}=T_{1}+C_{1} X_{1}^{(1)}\left(x, x_{1}\right)-\frac{q}{2} X_{1}^{(2)}\left(x, x_{1}\right), & x_{1} \leqslant x \leqslant x_{2}, \\
T^{(2)}=T_{\phi}+C_{2} X_{2}^{(1)}\left(x, x_{2}\right)-\frac{q}{2} X_{2}^{(2)}\left(x, x_{2}\right), & x_{2} \leqslant x \leqslant x_{3}, \\
T^{(3)}=T_{4}+C_{3} X_{3}^{(1)}\left(x, x_{4}\right)-\frac{q}{2} X_{3}^{(2)}\left(x, x_{4}\right), & x_{3} \leqslant x \leqslant x_{4},
\end{array}
$$

где $T_{\phi}$ - температура фазового перехода вещества слоя, $T_{1}, T_{4}-$ - температуры на внешних границах, выражение вида $X^{(1)}\left(x, x_{0}\right)$ обозначает первую обобщенную степень Берса с нуль-точкой 
$x_{0}$, а выражение вида $X^{(2)}\left(x, x_{0}\right)$-一 вторую обобщенную степень Берса. Номер слоя в обобщенных степенях записывается в нижнем индексе.

Приведем основные свойства обобщенных степеней Берса:

$$
\begin{aligned}
& X^{(1)}\left(x, x_{0}\right)=\int_{x_{0}}^{x} \frac{d \xi}{a_{1}(\xi)}, \\
& \tilde{X}^{(1)}\left(x, x_{0}\right)=\int_{x_{0}}^{x} \frac{d \xi}{a_{2}(\xi)},
\end{aligned}
$$

где $a_{1}(x), a_{2}(x)$ - положительные функции, непрерывные на заданном промежутке,определенные физическими и геометрическими параметрами среды. Для процесса теплопроводности, заданного уравнением $(1)$, функции $a_{1}^{(i)}(x)$ и $a_{2}^{(i)}(x)$ определяются соотношениями

$$
a_{1}^{(i)}=\lambda^{(i)} x^{s}, \quad a_{2}^{(i)}=x^{-s},
$$

где $\lambda^{(i)}$ - коэффициент теплопроводности $i$-го слоя. Значение показателя $s$ определяется геометрией среды: значение $s=0$ соответствует процессу теплопроводности в среде со сдвиговой симметрией (плоские слои) по оси $x, s=1$-с осевой симметрией (цилиндрические слои) и $s=2-$ с центральной симметрией (сферические слои). Далее,

$$
\begin{gathered}
X^{(2)}\left(x, x_{0}\right)=2 \int_{x_{0}}^{x} \frac{\tilde{X}^{(1)}\left(\xi, x_{0}\right) d \xi}{a_{1}(\xi)}, \\
X^{(1)}\left(x_{0}, x_{0}\right)=0, \quad \tilde{X}^{(1)}\left(x_{0}, x_{0}\right)=0, \quad X^{(2)}\left(x_{0}, x_{0}\right)=0, \\
D_{1}=a_{1} \frac{d}{d x}, \quad D_{1}=a_{2} \frac{d}{d x}, \\
D_{1} X^{(1)}\left(x, x_{0}\right)=1, \quad D_{2} \tilde{X}^{(1)}\left(x, x_{0}\right)=1, \quad D_{1} X^{(2)}\left(x, x_{0}\right)=2 \tilde{X}^{(1)}\left(x, x_{0}\right), \\
J=-D_{1} T(x),
\end{gathered}
$$

где $J$ - плотность потока тепла.

За счет выбора нуль-точек в обобщенных степенях Берса удовлетворены внешние граничные условия

$$
T^{(1)}\left(x_{1}\right)=T_{1}, \quad T^{(3)}\left(x_{4}\right)=T_{4},
$$

а также значение $T^{(2)}\left(x_{2}\right)$, равное $T_{\phi}$, полученное как предел справа. Таким образом, имеем два условия для определения $x_{2}, x_{3}$ :

$$
\begin{aligned}
& C_{1} X_{1}\left(x_{2}, x_{1}\right)-\frac{q}{2} X_{1}^{(2)}\left(x_{2}, x_{1}\right)=T_{\phi}-T_{1}, \\
& C_{3} X_{3}\left(x_{3}, x_{4}\right)-\frac{q}{2} X_{3}^{(2)}\left(x_{3}, x_{4}\right)=T_{\phi}-T_{4} .
\end{aligned}
$$

Предложенный метод приводит к алгебраическим уравнениям в виде двух многочленов от $x_{2}, x_{3}$ степени не выше трех. Очевидно, их можно решить графически, изобразив систему на плоскости $x_{2}, x_{3}$. Такой способ решения напоминает обратное интерполирование (см. [1]).

Далее рассматриваем только плоскую оболочку. Задача значительно упрощается при симметрии граничных условий, т.е. $T_{1}=T_{4}$. Решение, очевидно, имеет плоскость симметрии при $\left(x_{1}+x_{4}\right) / 2=x_{c}$, а $x_{3}=x_{4}+x_{1}-x_{2}$. При $x=x_{c}$ в силу симметрии поток $J$ равен нулю.

Учитывая эти условия, имеем краевую задачу со смешанными граничными условиями:

$$
T^{(1)}\left(x_{1}\right)=T_{1}=T_{4},\left.\quad \frac{d T^{(1)}}{d x}\right|_{x=x_{c}}=0 .
$$

В силу симметрии граничных условий можем перейти к упрощенной модели оболочки из двух слоев (рис. 2). 
(1)

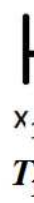

(2)

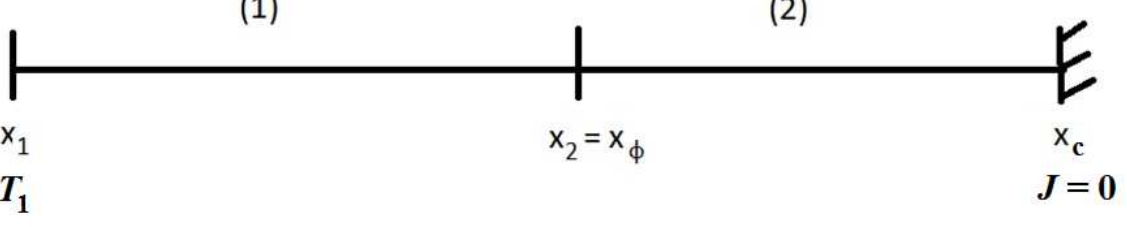

Рис. 2. Упрощенная модель из двух слоев с учетом симметрии граничных условий, описывающая фазовые переходы в плоской оболочке

Решение ищем в виде

$$
\begin{array}{ll}
T^{(1)}(x)=T_{1}+C_{1} X_{1}^{(1)}\left(x, x_{1}\right)-\frac{q}{2} X_{1}^{(2)}\left(x, x_{1}\right), & x_{1} \leqslant x \leqslant x_{2}, \\
T^{(1)}(x)=T_{\phi}+C_{2} X_{2}^{(1)}\left(x, x_{2}\right)-\frac{q}{2} X_{2}^{(2)}\left(x, x_{2}\right), & x_{2} \leqslant x \leqslant x_{c},
\end{array}
$$

при граничных условиях

$$
T^{(1)}\left(x_{1}\right)=T_{1},\left.\quad \frac{d T^{(2)}(x)}{d x}\right|_{x=x_{c}}=0
$$

и условиях согласования в точке $x_{2}=x_{\phi}$

$$
T^{(1)}\left(x_{2}\right)=T^{(2)}\left(x_{2}\right)=T_{\phi},\left.\quad D_{1}^{(1)} T^{(1)}(x)\right|_{x=x_{2}}=\left.D_{1}^{(2)} T^{(2)}(x)\right|_{x=x_{2}} .
$$

Имеем пять условий на решение: два граничных условия, два условия согласования и одно условие определенного значения $T\left(x_{2}\right)=T_{\phi}$ в точке $x_{2}=x_{\phi}$ границы фаз. Из граничного условия отсутствия потока в точке $x_{c}$ и $(6)$ имеем

$$
C_{2}=\tilde{X}_{2}^{(1)}\left(x_{c}, x_{2}\right),
$$

а из условия согласования по потоку в точке $x_{2}$

$$
C_{1}-\tilde{X}_{1}^{(1)}\left(x_{2}, x_{1}\right)=C_{2}-\tilde{X}_{2}^{(1)}\left(x_{2}, x_{2}\right),
$$

откуда для $C_{1}$ получим

$$
C_{1}=\tilde{X}_{1}^{(1)}\left(x_{2}, x_{1}\right)+\tilde{X}_{2}^{(1)}\left(x_{c}, x_{2}\right) .
$$

Подставляя $C_{1}$ в $(5)$ и учитывая соотношение $T^{(1)}\left(x_{2}\right)=T_{\phi}$, получим следующее выражение для определения координаты $x_{2}=x_{\phi}$ :

$$
X_{1}^{(2)}\left(x_{\phi}, x_{1}\right)-2 X_{1}^{(1)}\left(x_{\phi}, x_{1}\right)\left(\tilde{X}^{(1)}\left(x_{\phi}, x_{1}\right)+\tilde{X}_{2}^{(1)}\left(x_{c}, x_{\phi}\right)\right)+\frac{2}{q}\left(T_{\phi}-T_{1}\right)=0 .
$$

Подставив выражения обобщенных степеней для плоского слоя и положив $x_{1}=0$, получим квадратное уравнение

$$
x_{\phi}^{2}-2 x_{c} x_{\phi}+\frac{2 \lambda_{1}}{q}\left(T_{\phi}-T_{1}\right)=0 .
$$

Таким образом, координата точки фазового перехода

$$
x_{\phi}=x_{c} \pm \sqrt{x_{c}^{2}-\frac{2 \lambda}{q}\left(T_{\phi}-T_{1}\right)} .
$$


3. Матричный метод. Более удобно для решения поставленной задачи использовать матричный метод, описанный ранее в [3-5]. Чтобы охватить единым подходом случаи осесимметричной и центрально-симметричной системы слоев, используем аппарат обобщенных степеней Берса. Рассматриваемый матричный метод можно применять при любом конечном числе слоев.

Введем для $k$-го слоя $\left[x_{k}, x_{k+1}\right]$ вектор-столбцы $V, W$ и матрицу $K$ следующим образом:

$$
\begin{gathered}
V^{(1)}\left(x_{1}\right)=\left(\begin{array}{c}
T^{(1)}\left(x_{1}\right) \\
J^{(1)}\left(x_{1}\right)
\end{array}\right), \quad V^{(k)}(x)=\left(\begin{array}{c}
T^{(k)}(x) \\
J^{(k)}(x)
\end{array}\right), \quad W^{(k)}\left(x, x_{k}\right)=\left(\begin{array}{c}
w^{(k)}(x)-w^{(k)}\left(x_{k}\right) \\
-D^{(k)} w^{(k)}
\end{array}\right), \\
K^{(k)}\left(x, x_{k}\right)=\left(\begin{array}{cc}
1 & -X_{k}^{(1)}\left(x, x_{k}\right) \\
0 & 1
\end{array}\right) .
\end{gathered}
$$

Здесь $J^{(k)}$ - плотность потока в $k$-м слое:

$$
J^{(k)}(x)=-\lambda^{(k)} D_{1}^{(k)} T^{(k)}(x),
$$

а функции $w^{(k)}(x)$ - произвольные частные решения неоднородных уравнений (1).

Для $w^{(k)}(x)$ выберем

$$
w^{(k)}(x)=-\frac{q}{2} X^{(2)}\left(x, x_{k}\right) .
$$

Решение задачи Коши при заданных $T^{(k)}\left(x_{k}\right), J^{(k)}\left(x_{k}\right)$ имеет вид

$$
V^{(k)}(x)=\left(\begin{array}{l}
T^{(k)}(x) \\
J^{(k)}(x)
\end{array}\right)=K^{(k)}\left(x, x_{k}\right) V^{(k)}\left(x_{k}\right)+W^{(k)}\left(x, x_{k}\right) .
$$

Решение для всех трех слоев, определяющих фазы в слое, запишем в виде

$$
\begin{aligned}
& V^{(1)}(x)=K^{(1)}\left(x, x_{1}\right) V^{(1)}(1)+W^{(1)}\left(x, x_{1}\right), \quad x_{1} \leqslant x \leqslant x_{2}, \\
& V^{(2)}(x)=K^{(2)}\left(x, x_{2}\right) K^{(1)}\left(x_{2}, x_{1}\right) V^{(1)}(1)+ \\
& +K^{(2)}\left(x, x_{2}\right) W^{(1)}\left(x_{2}, x_{1}\right)+W^{(2)}\left(x, x_{2}\right), \quad x_{2} \leqslant x \leqslant x_{3}, \\
& V^{(3)}(x)=K^{(3)}\left(x, x_{3}\right) K^{(2)}\left(x_{3}, x_{2}\right) K^{(1)}\left(x_{2}, x_{1}\right) V^{(1)}(1)+ \\
& +K^{(3)}\left(x, x_{3}\right) K^{(2)}\left(x_{3}, x_{2}\right) W^{(2)}\left(x_{2}, x_{1}\right)+ \\
& +K^{(3)}\left(x, x_{3}\right) W^{(2)}\left(x_{3}, x_{2}\right)+W^{(3)}\left(x, x_{3}\right), \quad x_{3} \leqslant x \leqslant x_{4} .
\end{aligned}
$$

Подставляя $x=x_{4}$ и $T\left(x_{4}\right)=T_{4}$ в $(9)$, находим значение $J^{(1)}\left(x_{1}\right)$, выраженное через искомые $x_{2}$ и $x_{3}$.

Подставим $J^{(1)}\left(x_{1}\right)$ в $(7)$ и в $(8)$, после чего из системы

$$
T\left(x_{2}\right)=T_{\phi}, \quad T\left(x_{3}\right)=T_{\phi}
$$

найдем границы фаз $x_{2}$ и $x_{3}$.

Описанный метод пригоден для систем слоев со сдвиговой, осевой или центральной симметрией. Применяя матричный метод к системе трех слоев при симметричных граничных условиях, получаем результат, который совпадает с полученным выше при помощи традиционного способа решения. Обладая большой общностью, матричный метод позволяет рассмотреть системы с любым конечным числом слоев, расположенных слева и справа от слоя с фазовым переходом. Решение задачи лежит в классе непрерывных функций,имеющих конечное число разрывов производной. Метод допускает различные обобщения по усложнению граничных условий и условий согласования.

4. Заключение. В работе предложен аналитический метод нахождения границ фазового перехода в многослойной среде с распределенными источниками тепла. Представленный подход с использованием аппарата обобщенных степеней Берса и матричного метода допускает обобщения для различной геометрии многослойной среды и усложнению граничных условий и условий согласования. 


\section{СПИСОК ЛИТЕРАТУРЫ}

1. Вержбицкий В. М. Численные методы. Математический анализ и обыкновенные дифференциальные уравнения. - М.: Высшая школа, 2001.

2. Гладышев Ю. А. Метод обобщенных степеней Берса и его приложение в математической физике. Калуга: КГУ им. К. Э. Циолковского, 2011.

3. Гладышев Ю. А., Дворянчикова Ю. В. Процесс теплопроводности в неоднородной пластине при переменном коэффициенте внешнего теплообмена// в кн.: 5 Российская национальная конференция по теплообмену. - М.: МЭИ, 2010. - С. 76-79.

4. Гладышев Ю. А., Калманович В. В., Серегина Е. В., Степович М. А. О возможности совместного применения матричного метода и аппарата обобщенных степеней Берса для математического моделирования процесса теплопереноса в объектах, обладающих цилиндрической симметрией// Вопросы атомной науки и техники. Сер. Ядерно-реакторные константы. - 2018. - № 3. - С. 158-167.

5. Калманович B. B., Степович M. А. О совместном применении матричного метода и аппарата обобщенных степеней Берса для математического моделирования процессов тепломассопереноса в полупроводниковых материалах электронной техники// в кн.: Проблемы разработки перспективных микро- и наноэлектронных систем. - М.: ИППМ РАН, 2018. - С. 194-201.

6. Кудинов В. А., Карташов Э. М., Калашников В. В. Аналитические решения задач тепломассопереноса и термоупругости для многослойных конструкций. - М.: Высшая школа, 2005.

7. Bers L., Gelbart A. On a class of functions defined by partial differential equations// Trans. Am. Math. Soc. - 1944. - 56. - P. 67-93.

Афанасенкова Юлия Вячеславовна

Калужский государственный университет им. К. Э. Циолковского

E-mail: dvoryanchikova_y@mail.ru

Гладышев Юрий Александрович

Калужский государственный университет им. К. Э. Циолковского

E-mail: v572264@yandex.ru

Калманович Вероника Валерьевна

Калужский государственный университет им. К. Э. Циолковского

E-mail: v572264@yandex.ru 